International Journal of Pure and Applied Mathematics

Volume 115 No. $1 \quad 2017,123-127$

ISSN: 1311-8080 (printed version); ISSN: 1314-3395 (on-line version)

url: http://www.ijpam.eu

doi: 10.12732/ijpam.v115i1.10

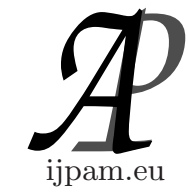

\title{
T-SEPERATING SETS FOR COHERENT SEQUENCES
}

\author{
Martin Dowd \\ 60 Mooring Ln. \\ Daly City, CA 94014, USA
}

\begin{abstract}
In a previous paper the author used methods of Witzany to give a lower bound for the smallest repeat point of a coherent sequence. Here the notion of a T-seperating set is introduced, and the lower bound is improved.
\end{abstract}

AMS Subject Classification: 03E55

Key Words: coherent sequence, repeat point

\section{Introduction}

In [4] some methods are introduced for constructing seperating stationary sets for coherent sequences of normal ultrafilters. Some further results are given in [3]. Here these methods are improved on. Superschemes were introduced in [1], and an improved discussion is given in [2]. The methods here permit the use of superschemes in constructing seperating stationary sets.

As noted in [3], by results of Mitchell there is a model $L[\mathcal{U}]$ such that in $L[\mathcal{U}], \mathcal{U}$ is a coherent sequence of normal ultrafilters comprising all the norml ultrafilters. It is well-known that GCH holds in $L[\mathcal{U}]$.

Notation for coherent sequences will be as in [3]. Hereafter in this section it will be assumed that GCH holds and $\mathcal{U}$ is maximal, so that for a measurable cardinal $\kappa, \operatorname{Dom}(\mathcal{U}(\kappa))=o(\kappa) \leq \kappa^{++}$.

\section{Seperating Sets}

Say that $S$ is a seperating set for $\mathcal{U}(\kappa)$ at $\alpha$ if $S \in \mathcal{U}(\kappa)(\alpha)$ but $S \notin \mathcal{U}(\kappa)(\beta)$

$\begin{array}{lcc}\text { Received: } & \text { May 8, } 2017 & \text { (C) } 2017 \text { Academic Publications, Ltd. } \\ \text { Revised: } & \text { June 3, } 2017 & \text { url: www.acadpubl.eu } \\ \text { Published: } & \text { June 28, } 2017 & \end{array}$


for $\beta<\alpha$; such exists iff $\alpha$ isnot a repeat point. Say that $S$ is T-seperating if in addition $S \in \mathcal{U}(\kappa)(\beta)$ for $\alpha \leq \beta<\operatorname{Dom}(\mathcal{U}(\kappa))$. These may readily be seen to exist for $\alpha$ up to a bound given in theorem 22.g of [3].

Given a measurable cardinal $\kappa$ and a function $f: \kappa \mapsto$ Ord let $D_{f}^{\geq}=\{\lambda \in$ Card $\cap \kappa: o(\lambda) \geq f(\lambda)$. For $\beta<\operatorname{Dom}(\kappa), U_{\beta}$ will be used as an abbreviation for $\mathcal{U}(\kappa)(\beta)$.

Theorem 1. Suppos $\alpha<\operatorname{Dom}(\mathcal{U}(\kappa))$ and $f$ represents $\alpha$ on $[0, \operatorname{Dom}(\mathcal{U}(\kappa)))$. Then $D_{f}^{\geq}$is T-seperating at $\alpha$.

Proof. $D_{f}^{\geq} \in U_{\beta}$ iff $[o]_{U_{\beta}} \geq[f]_{U_{\beta}}$ iff $\beta \geq \alpha$.

It may be easier to construct T-seperating sets than representing functions on $[0, \operatorname{Dom}(\mathcal{U}(\kappa)))$, and studying them in their own right is of interest.

Theorem 2. Suppose $S$ is a seperating set at $\alpha$ and $\alpha+1<\operatorname{Dom}(\mathcal{U}(\kappa))$. Then there is a $T$-seperating set $S^{\prime}$ at $\alpha+1$.

Proof. Let $S^{\prime}=\{\lambda \in \operatorname{Card} \cap \kappa: \exists \eta<\operatorname{Dom}(\mathcal{U}(\lambda))(S \cap \lambda \in \mathcal{U}(\lambda)(\eta))$. Using coherence and the fact that $S$ is seperating, $S^{\prime} \in U_{\beta}$ iff $\models_{\mathrm{Ult}_{U_{\beta}}(V)} \exists \eta<$ $\operatorname{Dom}(\mathcal{U}(\kappa))(S \in \mathcal{U}(\kappa)(\eta))$ iff $\beta>\alpha$.

Theorem 3. Suppose $\eta<\kappa$, for $\xi<\eta S_{\xi}$ is a T-seperating set at $\alpha_{\xi}$, $\alpha=\sup _{\xi<\eta} \alpha_{\xi}$, and $\alpha<\operatorname{Dom}(\mathcal{U}(\kappa))$. Let $S=\cap_{\xi<\eta} S_{\xi}$; then $S$ is a T-seperating set at $\alpha$.

Proof. For $\beta \geq \alpha$, since $S_{\xi} \in U_{\beta}$ for $\xi<\eta$ and $U_{\beta}$ is $\kappa$-complete, $S \in U_{\beta}$. If $\beta<\alpha$ then $\beta<\alpha_{\xi}$ for some $\xi$, so $S_{\xi} \notin U_{\beta}$. so $S \notin U_{\beta}$ since $S \subseteq S_{\xi}$.

Theorem 4. Suppose for $\xi<\kappa S_{\xi}$ is a T-seperating set at $\alpha_{\xi}, \alpha=$


at $\alpha$.

Proof. For $\beta \geq \alpha$, since $S_{\xi} \in U_{\beta}$ for $\xi<\kappa$ and $U_{\beta}$ is normal, $S \in U_{\beta}$. If $\beta<\alpha$ then $\beta<\alpha_{\xi}$ for some $\xi$, so $S_{\xi} \notin U_{\beta}$. so $S \notin U_{\beta}$ since $S \subseteq_{\mathcal{I}} S_{\xi}$ where $\mathcal{I}$ is the thin ideal.

Recall from [2] that for $\kappa \in$ Card a scheme is a pair $\Sigma=\langle\sigma, \phi\rangle$ where $\sigma<\kappa^{+}$and $\phi$ is a function whose domain is the set of limit ordinals $\alpha \leq \sigma$. For $\alpha \in \operatorname{Dom}(\phi), \phi(\alpha)$ is an increasing function with domain an ordinal $\eta \leq \kappa$, and whose range is an unbounded subset of $\alpha$. If $\operatorname{cf}(\alpha)<\kappa$ then $\eta<\kappa$, and if $\operatorname{cf}(\alpha)=\kappa$ then $\eta=\kappa$. 
A scheme is a recipe for an iteration. Given a scheme $\Sigma$ with $\sigma<\operatorname{Dom}(\kappa)$ the subset $S_{\alpha}^{\Sigma}$ may be defined inductively for $\alpha \leq \sigma$ as follows.

0. $S_{0}^{\Sigma}=\operatorname{Card} \cap \kappa$.

1. $S_{\beta+1}^{\Sigma}=\left(S_{\beta}^{\Sigma}\right)^{\prime}$, as in theorem 2 .

2. $(\alpha \in \operatorname{Lim}, \operatorname{cf}(\alpha)<\kappa) \cap_{\xi<\operatorname{Dom}(\phi(\alpha))} S_{\phi(\alpha)(\xi)}^{\Sigma}$.

3. $(\alpha \in \operatorname{Lim}, \operatorname{cf}(\alpha)=\kappa) \triangle \xi<\kappa S_{\phi(\alpha)(\xi)}^{\Sigma}$.

By theorems 2-4 $\Sigma_{\alpha}^{\Sigma}$ is T-seperating for all $\alpha \leq \sigma$.

Suppose there is an $\alpha$ there is no T-seperating set at $\alpha$, and let $\theta$ be the least such $\alpha$. For another corollary of theorems $2-4, \operatorname{cf}(\theta)=\kappa^{+}$.

\section{Superschemes}

Recall from [2] that for $\kappa \in$ Card a superscheme is a pair $\Sigma=\langle\sigma, \phi\rangle$ where $\sigma<\kappa^{++}$and $\phi$ is as for a scheme. To use a superscheme for an iteration a method must be specified for obtaining $S_{\alpha}$ when $\operatorname{cf}(\alpha)=\kappa^{+}$. Some discussion of this problem will be given here.

Suppose $\alpha=\kappa^{+}, U$ is a normal ultrafilter on $\kappa$, and $M=\operatorname{Ult}_{U}(V)$. Since the well-orders of $\kappa$, coded as subsets of $\kappa$, are the same in $V$ and $M$, and the order type function is $\Delta^{1},\left(\kappa^{+}\right)^{M}=\kappa^{+}$. Thus, the function $\lambda \mapsto \lambda^{+}$represents $\kappa^{+} \bmod$ any normal ultrafilter on $\kappa$, and a T-seperating set may be constructed using theorem 1 .

Alternatively, referring to [3] let $\preceq$ be a $\Delta_{\infty}^{1}$ WPS with $\Omega(\prec)=\kappa^{+}$(in fact there is a $\Sigma_{1}^{1} \preceq$ ). According to theorem 10 of $[3], \lambda \mapsto \Omega\left(\prec_{\lambda}\right)$ (defined on $D_{\preceq}$ ) represents $\kappa^{+}$in any $\operatorname{Ult}_{U}(V)$.

Another construction may be given by adapting a method of proposition 3.9 if [4]. As in [3] let $C$ denote the map $\alpha \mapsto j_{\mathcal{U}(\kappa)(\alpha)}(\kappa) . C_{\mathcal{U}(\kappa)}$ may be written to indicate $\mathcal{U}$ and $\kappa$.

Theorem 5. Supopse $S$ is a seperating set at $\alpha$. For any $\beta \in(\alpha$, $\min (\operatorname{Dom}(\mathcal{U}(\kappa)), C(\alpha)))$ there is a $T$-seperating set at $\beta$.

Proof. As in the proof of theorem 2 let $S_{1}=\{\lambda \in \operatorname{Card} \cap \kappa: \exists \eta<$ $\operatorname{Dom}(\mathcal{U}(\lambda))(S \cap \lambda \in \mathcal{U}(\lambda)(\eta))\}$, so that $S_{1} \in U_{\beta}$ iff $\beta>\alpha$, For $\lambda \in S_{1}$ let $f_{\alpha}(\lambda)$ be the least $\eta$; then for $\beta>\alpha$ the function $f \alpha$ represents $\alpha$ in $\operatorname{Ult}(\kappa)(\beta)$. Choose $g_{n} u: \kappa \mapsto \kappa$ such that $\left[g_{\beta}\right]_{\mathcal{U}(\kappa)(\alpha)}=\beta$. For $\lambda \in S_{1}$ let $\tilde{g}(\lambda)=\left[g\lceil\lambda]_{\mathcal{U}(\lambda)\left(f_{\alpha}(\lambda)\right)}\right.$; otherwise let $\tilde{g}(\lambda)=0$. Let $M$ denote $\mathcal{U}(\kappa)(\beta)$. Then $g \in M$, and one verifies (see [4]) that $[\tilde{g}]_{\mathcal{U}}(\kappa)(\beta)=\beta$. The set $S_{1} \cap D_{\tilde{g}}^{\geq}$is T-seperating at $\beta$.

Let $C^{(1)}$ denote the fixed point enumerator of $C$. If there is an $\alpha$ such that 
there is no T-seperating set at $\alpha$ let $\theta$ denote the smallest such. As in theorem 22 of [3], $C(\theta)=\theta$ and $C^{(1)}\left(\kappa^{+}\right) \leq \theta$.

\section{Iterating $C$}

Let $C^{(\sigma)}$ denote the result of applying the fixed point operator to $C \sigma$ times. If $\operatorname{Dom}(\mathcal{U}(\kappa))=\kappa^{++}$then for $\sigma<\kappa^{++} \operatorname{Ran}\left(C^{(\sigma)}\right)$ is a club set, and the intersection of all these is empty. If $\operatorname{Dom}(\mathcal{U}(\kappa))<\kappa^{++}$then $\operatorname{Dom}\left(C^{(\sigma)}\right)$ decreases as $\sigma$ increases, becoming $\emptyset$ for some $\sigma \leq \operatorname{Dom}(\mathcal{U}(\kappa))$.

Theorem 6. Suppose $\alpha<\operatorname{Dom}(\mathcal{U}(\kappa)), f_{\alpha}$ represents $\alpha$ on $[0, \operatorname{Dom}(\mathcal{U}(\kappa)))$, and $\alpha \in \operatorname{Dom}\left(C^{(1)}\right)$. Then there is a $T$-seperating set at $C_{\mathcal{U}(\kappa)}^{(1)}(\alpha)$.

Proof. Let $S=\left\{\lambda: o(\lambda) \geq C_{\mathcal{U}(\lambda)}^{(1)}\left(f_{\alpha}(\lambda)\right)\right\}$. Then $S \in U_{\beta}$ iff $\beta \geq C_{\mathcal{U}(\kappa)}^{(1)}(\alpha)$. The condition on $\lambda$ holds iff either $\exists \eta<\operatorname{Dom}(\mathcal{U}(\lambda))\left(\eta=C_{\mathcal{U}(\lambda)}^{(1)}\left(f_{\alpha}(\lambda)\right)\right)$, or $\operatorname{Dom}(\mathcal{U}(\lambda))$ is a limit ordinal and $\forall \zeta<f_{\alpha}(\lambda) \exists \eta<\operatorname{Dom}(\mathcal{U}(\lambda))\left(\eta=C_{\mathcal{U}(\lambda)}^{(1)}(\zeta)\right)$ and $\forall \zeta<\operatorname{Dom}(\mathcal{U}(\lambda))\left(C_{\mathcal{U}(\lambda)}(\zeta)<\operatorname{Dom}(\mathcal{U}(\lambda))\right)$.

Theorem 7. Supoose $\alpha<\operatorname{Dom}(\mathcal{U}(\kappa))$, there is a T-seperating set at $\alpha$, and $C_{\mathcal{U}(\kappa)}^{(1)}(\alpha)<\operatorname{Dom}(\mathcal{U}(\kappa))$. Then there is a T-seperating set at $C_{\mathcal{U}(\kappa)}^{(1)}(\alpha)$.

Proof. The proof of theorem 6 may be modified. Let $S_{1}$ be a T-seperating set at $\alpha$. Let $f_{\alpha}^{E}$ be an E-representing function for $\alpha$, as defined in [3]. Let $S_{2}$ be the set $S$, as in the proof of theorem 6 , with $f_{\alpha}^{E}$ used in place of $f_{\alpha}$. Then $S_{1} \cap S_{2}$ is T-seperating at $C^{(1)}(\alpha)$.

Theorem 8. Suppose $\alpha<\operatorname{Dom}(\mathcal{U}(\kappa)), f_{\alpha}$ represents $\alpha$ on $[0, \operatorname{Dom}(\mathcal{U}(\kappa)))$, $f_{\sigma}$ represents $\sigma$ on $[0, \operatorname{Dom}(\mathcal{U}(\kappa)))$, and $\alpha \in \operatorname{Dom}\left(C^{(\sigma)}\right)$. Then there is a $T$ seperating set at $C_{\mathcal{U}(\kappa)}^{(\sigma)}(\alpha)$.

Proof. Let $S=\left\{\lambda: o(\lambda) \geq C_{\mathcal{U}(\lambda)}^{\left(f_{\sigma}(\lambda)\right)}\left(f_{\alpha}(\lambda)\right)\right\}$. Then $S \in U_{\beta}$ iff $\beta \geq C_{\mathcal{U}(\kappa)}^{(\sigma)}(\alpha)$. The condition on $\lambda$ holds iff either $\exists \eta<\operatorname{Dom}(\mathcal{U}(\lambda))\left(\eta=C_{\mathcal{U}(\lambda)}^{(\sigma)}\left(f_{\alpha}(\lambda)\right)\right)$, or $\operatorname{Dom}(\mathcal{U}(\lambda))$ is a limit ordinal and $\forall \zeta<f_{\alpha}(\lambda) \exists \eta<\operatorname{Dom}(\mathcal{U}(\lambda))\left(\eta=C_{\mathcal{U}(\lambda)}^{\left(f_{\sigma}(\lambda)\right)}(\zeta)\right)$ and $\forall \zeta<\operatorname{Dom}(\mathcal{U}(\lambda)) \forall \tau<f_{\sigma}(\lambda)\left(C_{\mathcal{U}(\lambda)}^{(\tau)}(\zeta)<\operatorname{Dom}(\mathcal{U}(\lambda))\right)$.

Theorem 9. Supoose $\alpha<\operatorname{Dom}(\mathcal{U}(\kappa))$, there is a $T$-seperating set at $\alpha$, there is a T-seperating set at $\sigma$, and $\alpha \in \operatorname{Dom}\left(C^{(\sigma)}\right)$. Then there is a T-seperating set at $C_{\mathcal{U}(\kappa)}^{(\sigma)}(\alpha)$. 
Proof. The proof of theorem 8 may be modified. Let $S_{1 \alpha}$ be a T-seperating set at $\alpha$. Let $f_{\alpha}^{E}$ be an E-representing function for $\alpha$. Let $S_{1 \sigma}$ be a T-seperating set at $\sigma$. Let $f_{\sigma}^{E}$ be an E-representing function for $\sigma$. Let $S_{2}$ be the set $S$, as in the proof of theorem 8, with $f_{\alpha}^{E}$ used in place of $f_{\alpha}$ and $f_{\sigma}^{E}$ used in place of $f_{\sigma}$. Then $S_{1 \alpha} \cap S_{1 \sigma} \cap S_{2}$ is T-seperating at $C^{(1)}(\alpha)$.

These methods can be pursued further. Whether there are methods which may be pursued in $L[\mathcal{U}]$ is a question of considerable interest.

\section{References}

[1] M. Dowd, Iterating Mahlo's operation, Int. J. Pure Appl. Math., 9, no. 4 (2003), 469-512, http://www.hyperonsoft.com/imol.pdf

[2] M. Dowd, Improved results in scheme theory, Int. J. Pure Appl. Math., 76, No. 2 (2012), 173-190, http://ijpam.eu/contents/2012-76-2/3/3.pdf

[3] M. Dowd, Set chains mod the Pi-1-1 enforceable filte, Int. J. Pure Appl. Math., to appear, http://www.hyperonsoft.com/lbrp.pdf

[4] J. Witzany, Possible behaviours of the reflection ordering of stationary sets, J. Symbolic Logic 60 no. 2 (1995), 534-547, doi: 10.2307/2275849. 
